\title{
The Amplitude Mode in the Quantum Phase Model
}

\author{
S.D. Huber, ${ }^{1}$ B. Theiler, ${ }^{1}$ E. Altman, ${ }^{2}$ and G. Blatter ${ }^{1}$ \\ ${ }^{1}$ Theoretische Physik, ETH Zurich, CH-8093 Zurich, Switzerland \\ ${ }^{2}$ Department of Condensed Matter Physics, The Weizmann Institute of Science, Rehovot, 76100, Israel
}

(Dated: November 20, 2018)

\begin{abstract}
We derive the collective low energy excitations of the quantum phase model of interacting lattice bosons within the superfluid state using a dynamical variational approach. We recover the well known sound (or Goldstone) mode and derive a gapped (Higgs type) mode that was overlooked in previous studies of the quantum phase model. This mode is relevant to ultracold atoms in a strong optical lattice potential. We predict the signature of the gapped mode in lattice modulation experiments and show how it evolves with increasing interaction strength.
\end{abstract}

PACS numbers: 05.30.Jp, 03.75.Kk, 39.25+k

Interactions can have a dramatic influence on the properties of superfluids at low temperatures. In the most extreme case, such as lattice bosons at commensurate filling, interactions drive a quantum phase transition to an insulating phase [1]. But even inside the superfluid phase, interactions may greatly impact on basic properties such as the excitations, with the roton minimum in the spectrum of Helium providing a well known example. In this letter, we investigate the effect of increasing interaction strength on the collective modes in a superfluid of lattice bosons; the latter are usually modeled by the Bose-Hubbard model incorporating both hopping (parameter $t$ ) and local interactions $(U)$. The question has been brought into focus by experiments with ultracold atoms in optical lattices that were able to control the interaction strength and even drive a transition to the insulating state [2]. More recent experiments have probed the excitation spectrum of the superfluid in this strongly correlated regime by measuring the energy absorption rate in response to periodic lattice modulations. The interpretation of the lattice modulation experiments is complicated by the large magnitude of the perturbation and the presence of a confining potential [3]; nevertheless, the results are very suggestive of the existence of a gapped collective mode in the superfluid phase [4, 5], which is at the center of interest in the present work.

The Bose-Hubbard model generates two effective lowenergy field theories for the complex order-parameter field $\psi$, see Fig. 1 for weak interaction $U \bar{n} \ll t$, the effect of the lattice can be absorbed in an effective band mass and one arrives at the Galilean-invariant Gross-Pitaevskii theory (nonlinear Schrödinger equation; $\bar{n}$ is the mean filling per site). Quite remarkably, close to the superfluidinsulator transition at $U \sim t \bar{n}$, the combined action of the lattice and the interaction leads to a Lorentz-invariant critical theory (nonlinear Klein Gordon equation) [6]. The first-order time derivative in the Gross-Pitaevskii theory defines the density $\rho=|\psi|^{2}$ and hence any density mode is bound to the phase degree of freedom, resulting in the unique and well known sound (or Goldstone) mode. This differs from the Lorentz-invariant critical

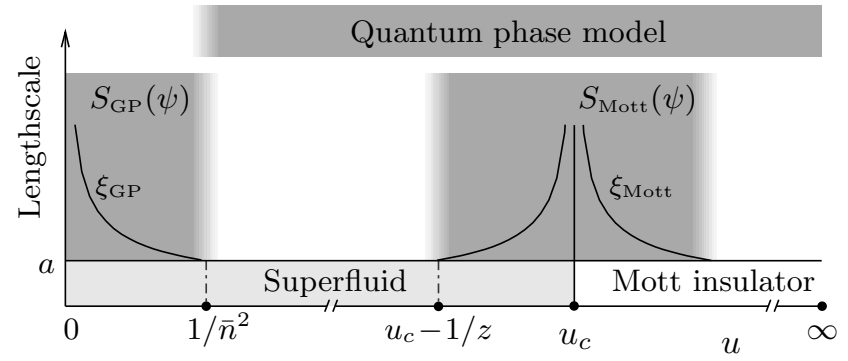

FIG. 1: Schematic overview of models describing the superfluid phase of lattice Bosons. For small (dimensionless) interactions $u=U / 2 t \bar{n} z<1 / \bar{n}^{2}$, the Bose-Hubbard model is well approximated by the continuum Gross-Pitaevskii action $S_{\mathrm{GP}}(\psi)$. Close to the critical interaction $u \approx u_{c}=2$, the dynamics is described by the critical theory $S_{\text {Mott }}(\psi)$. For $u>1 / \bar{n}^{2}$, the Bose-Hubbard model is equivalent to the quantum phase model, analyzed in detail in this work.

theory, where the second-order time derivative spoils the relation between the order-parameter modulus and the density; as a consequence, this theory admits the possibility of independent amplitude (Higgs) and phase (Goldstone) modes. Naturally, the question poses itself, how the Higgs mode emerges upon increasing the interaction $U$. This question should be addressed within a microscopic theory that explicitly accounts for the interplay between interactions and the lattice, as is done by the quantum phase model [7, 8], a suitable approximation of the Bose-Hubbard model for large filling $\bar{n}$. In what follows, we discuss in more detail the two effective lowenergy theories for the Bose-Hubbard model and their interrelation with the quantum phase model and then concentrate on the derivation of the amplitude mode and its experimental observation.

We start with the Bose-Hubbard model in a coherent 
state formulation where the action is given by

$$
\begin{aligned}
S_{\mathrm{BH}}\left(\left\{\psi_{i}\right\}\right)= & \int_{0}^{\hbar \beta} d \tau \mathcal{L}_{\mathrm{BH}}\left(\left\{\psi_{i}\right\}\right)=\int_{0}^{\hbar \beta} d \tau\left[\sum_{i} \psi_{i}^{*}\left(\hbar \partial_{\tau}-\mu\right) \psi_{i}\right. \\
& \left.-t \sum_{\langle i, j\rangle} \psi_{i}^{*} \psi_{j}+\frac{U}{2} \sum_{i}\left|\psi_{i}\right|^{2}\left(\left|\psi_{i}\right|^{2}-1\right)\right] ; \quad(1)
\end{aligned}
$$

here, $\mu$ is the chemical potential and $\beta=1 / k_{\mathrm{B}} T$ is the inverse temperature. The bosonic fields $\psi_{i}$ denote the amplitude of particles in a Wannier state at site $i$.

The diverging correlation length $\xi_{\text {Mott }} \propto 1 / \sqrt{z\left(u_{c}-u\right)}$ near the (commensurate) superfluid-Mott-insulator transition at $u=U / 2 t \bar{n} z=2$ allows for a description in terms of a continuum critical theory $\mathcal{L}_{\text {Mott }}(\psi)=\left|\hbar \partial_{\tau} \psi\right|^{2} / J z+$ $J z\left(u_{c}-u\right)\left[\xi_{\text {Mott }}^{2}|\nabla \psi|^{2}-|\psi|^{2}\right]+U|\psi|^{4} / 2$, with $z=2 d$ the coordination number and $J=2 t \bar{n}$. The emergent (Higgs) mode involves a collective oscillation of the amplitude $|\psi|$ of the order parameter with a frequency that vanishes at the transition. Such oscillation of the order parameter is accompanied by a local change in the non-condensed fraction, leaving the local density unchanged. Furthermore, this mode is independent of the usual sound mode; this can be understood from the fact that the order parameter vanishes towards the transition while the density remains constant.

Clearly the gapped amplitude mode is absent in the opposite regime, of very weak interactions $u \ll 1 / \bar{n}^{2}$. In this case, the length scale set by the interaction is the well known healing length $\xi_{\mathrm{GP}}=a \sqrt{t / U \bar{n}} \gg a$. The fact that $\xi_{\mathrm{GP}}$ is much larger than the lattice constant $a$ allows for a continuum (Gross-Pitaevskii) description $\mathcal{L}_{\mathrm{GP}}(\psi)=$ $\psi^{*} \hbar \partial_{\tau} \psi+U \bar{n} \xi_{\mathrm{GP}}^{2}|\nabla \psi|^{2}+U|\psi|^{4} / 2$. The effective Galilean invariance ensures that at zero temperature there is no amplitude mode independent of first sound.

Comparing the regions of validity of the above coarse grained theories, see Fig. 1, we observe that these regimes are parametrically separated from each other for $\bar{n} \gg 1$. The absence of a diverging length scale in the intermediate regime $1 / \bar{n}^{2}<u<1$ renders the effect of the lattice relevant. As the Galilean invariance is explicitly broken, the existence of an amplitude mode cannot be ruled out. For large site occupancy, the Hubbard model in the intermediate (and strong) interaction regimes is equivalent to the (simpler) quantum phase model [7, 8]

$$
\hat{H}_{\mathrm{QPM}}=-J \sum_{\langle i, j\rangle} \cos \left(\hat{\varphi}_{i}-\hat{\varphi}_{j}\right)+\frac{U}{2} \sum_{i} \delta \hat{n}_{i}^{2},
$$

with the Josephson coupling $J=2 t \bar{n}$. The conjugate operators $\hat{\varphi}_{i}$ and $\delta \hat{n}_{j},\left[\hat{\varphi}_{i}, \delta \hat{n}_{j}\right]=i \hbar \delta_{i j}$, describe the local phase and deviation from mean filling, respectively. The derivation of $\hat{H}_{\mathrm{QPM}}$ from (1) involves an integration over density fluctuations under the assumption $\langle\delta \hat{n}\rangle / \bar{n} \ll 1$ [1], which is valid for $u \approx 1 / \bar{n}^{2}$, and subsequent (re-) quantization. Here we restrict our considerations to the case of integer filling $\bar{n} \in \mathbb{N}$.
We analyze the quantum phase model within a dynamical variational approach [10], which accounts for both phase and amplitude degrees of freedom and allows us to capture the low-energy physics of a depleted condensate near the Mott-insulator transition. We first derive the static properties in a mean-field approach and then include dynamics within a Gaussian approximation. Finally, we discuss the response of the system to an external lattice modulation, thereby connecting our findings with recent experiments [11]. Before proceeding, we note that a gapped excitation closely related to the one considered here has been identified by Cazalilla et al. [4] using a bosonization approach to a system with a strongly anisotropic optical lattice potential.

Our variational wave function has the Gutzwiller form

$$
\begin{aligned}
|\Psi\rangle & =\prod_{i} \sum_{n_{i}} f_{n_{i}}\left(\sigma_{i}, \varphi_{i}\right)\left|n_{i}\right\rangle \quad \text { with } \\
f_{n_{i}}\left(\sigma_{i}, \varphi_{i}\right) & =\frac{1}{\left(2 \pi \sigma_{i}\right)^{1 / 4}} e^{-\left(n_{i}-\bar{n}\right)^{2} / 2 \sigma_{i}} e^{i \bar{n} \varphi_{i}},
\end{aligned}
$$

where $\left|n_{i}\right\rangle$ is a particle-number state of the quantum phase model and $\delta \hat{n}_{i}\left|n_{i}\right\rangle=\left(n_{i}-\bar{n}\right)\left|n_{i}\right\rangle$. The orderparameter in the state given by (3) is $\psi_{i}=\left\langle\exp \left(-i \hat{\varphi}_{i}\right)\right\rangle=$ $e^{-1 / 4 \sigma_{i}} e^{-i \varphi_{i}}$, so that $\varphi_{i}$ and $\sigma_{i}$ determine phase and amplitude (fluctuations) of $\psi_{i}$, respectively. The wavefunction $|\Psi\rangle$ has a norm $\langle\Psi \mid \Psi\rangle=\prod_{i} \sum_{n_{i}} f_{n_{i}}^{*} f_{n_{i}}=$ $\prod_{i} \sum_{m} \exp \left[-\left(m \pi \sigma_{i}\right)^{2}\right]$ and is not properly normalized, a consequence of the discreteness of the particle numbers. This is not a problem as long as we stay away from the transition so that the particle number fluctuation is large $\left(\sigma_{i} \gg 1\right)$. We will concentrate on this regime.

The variational energy $\epsilon_{\mathrm{var}}=\left\langle\Psi\left|\hat{H}_{\mathrm{QPM}}\right| \Psi\right\rangle$ is given by

$$
\epsilon_{\mathrm{var}}=-J \sum_{\langle i, j\rangle} e^{-\left(\sigma_{i}^{-1}+\sigma_{j}^{-1}\right) / 4} \cos \left(\varphi_{i}-\varphi_{j}\right)+\frac{U}{4} \sum_{i} \sigma_{i}
$$

and is minimized for $\varphi_{i}=\varphi_{\mathrm{mf}} \equiv 0$ and $\sigma_{i}=\sigma_{\mathrm{mf}} \equiv 0$ in the Mott phase. In the superfluid phase $\sigma_{\mathrm{mf}} \neq 0$, leading to an order-parameter (or condensate fraction)

$$
\left|\psi_{0}\right|^{2} \equiv e^{-1 / 2 \sigma_{\mathrm{mf}}}=e^{2 W(-\sqrt{u / 16})},
$$

where $W(x)$ is the Lambert- $W$ function [12], cf. Fig. 2(b).

If this scheme is carried out blindly all the way to strong coupling, the transition to the Mott insulator appears as of first (rather than second) order. Besides a local minimum at $\sigma_{i}=0$, which is always present, a second minimum first appears at $u_{\star}=16 / e^{2} \approx 2.16$ (spinodal point). This spurious first order transition (at $u_{1} \approx 1.47$ ) is due to the failure of the variational wave-function when the particle number fluctuations become small $\left\langle\delta \hat{n}_{i}^{2}\right\rangle \ll 1$ near the transition [8]. However, as mentioned earlier we will use this approach only away from the critical regime of $\mathcal{L}_{\text {Mott }}(\psi)$ where (3) should be a good approximation. 


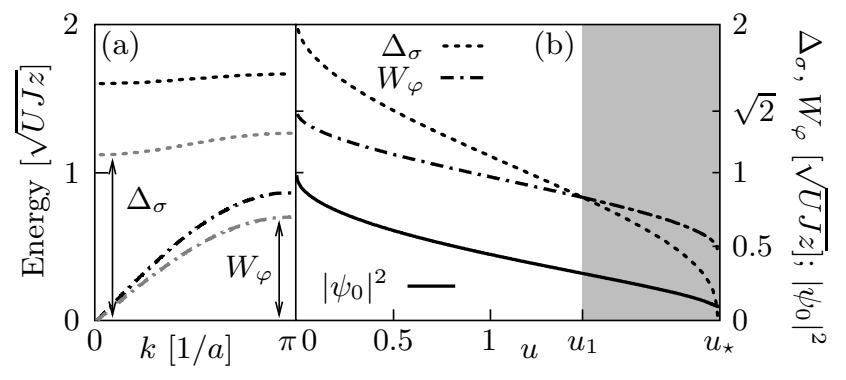

FIG. 2: (a) Spectra for the phonon (dash-dotted line) and the amplitude mode (dotted line). Black curves correspond to $u=U / J z=0.25$, gray curves to $u=1$. (b) Gap $\Delta_{\sigma}$ of the amplitude mode (dotted line) and the phonon bandwidth $W_{\varphi}$ (dash-dotted line) vs dimensionless coupling $u=U / J z$ in the superfluid phase. The gray shaded area corresponds to the Mott phase. The solid line denotes the condensate fraction $\left|\psi_{0}\right|^{2}$.

Next, we describe the fluctuations above the mean-field ground state (3) using a time-dependent variational principle. Following Ref. [10] we aim at an effective action (we switch to real time)

$$
S_{\text {eff }}=\int d t \mathcal{L}_{\text {eff }}=\int d t\left\langle\Psi\left|i \hbar \partial_{t}-\hat{H}_{\mathrm{QPM}}\right| \Psi\right\rangle
$$

While the minimizer of this expression provides the exact action in case of unrestricted and properly normalized variational states $|\Psi\rangle$, here, we restrict ourselves to the class of variational wavefunctions (cf. 31)

$$
f_{n_{i}}=\frac{1}{\left(2 \pi \sigma_{i}\right)^{1 / 4}} e^{-\left(n_{i}-\Phi_{i}\right)^{2}\left(1 / 2 \sigma_{i}-2 i \Sigma_{i}\right)} e^{i\left(n_{i}-\Phi_{i}\right) \varphi_{i}}
$$

This ansatz generates the Lagrangian

$$
\begin{aligned}
& \mathcal{L}_{\text {eff }}=\sum_{i} \hbar \dot{\sigma}_{i} \Sigma_{i}+\hbar \dot{\varphi}_{i} \Phi_{i}-\frac{U}{4} \sigma_{i}-\frac{U}{2}\left(\bar{n}-\Phi_{i}\right)^{2} \\
& +J \sum_{\langle i, j\rangle} e^{-\left(1+16 \Sigma_{i}^{2} \sigma_{i}^{2}\right) / 4 \sigma_{i}} e^{-\left(1+16 \Sigma_{j}^{2} \sigma_{j}^{2}\right) / 4 \sigma_{j}} \cos \left(\varphi_{i}-\varphi_{j}\right) .
\end{aligned}
$$

The new parameters $\Sigma_{i}$ and $\Phi_{i}$ in the wave function (7) assume the role of canonically conjugate fields and allow the order parameter's amplitude $\left(\sigma_{i}\right)$ and phase $\left(\varphi_{i}\right)$ degrees of freedom to acquire dynamics. The interaction terms $\propto U$ are linear/quadratic in these fields and the coupling between phase $\left(\varphi_{i}\right)$ and amplitude $\left(\sigma_{i}\right)$ degrees of freedom is only through the hopping term $\propto J$.

We obtain an effective action in terms of the fields $\sigma_{i}$ and $\varphi_{i}$ by solving the Euler-Lagrange equations for $\Sigma_{i}$ and $\Phi_{i}$. The static solution of the Euler-Lagrange equations for the $\sigma_{i}$ and $\varphi_{i}$ fields reproduces the meanfield ground state Eq. (5). Expanding the effective action around $\left(\sigma_{\mathrm{mf}}, \varphi_{\mathrm{mf}}\right)$ to second order, we find decoupled amplitude and phase degrees of freedom in $\mathcal{L}_{\text {eff }}$. We use a Legendre transformation $\left[\left(x_{i}, \dot{x}_{i}\right) \rightarrow\left(x_{i}, \Pi_{x_{i}}\right)\right.$, where $x=\sigma, \varphi]$ to quantize the real, classical fields introducing ladder operators $\left(\left[\hat{x}_{\mathbf{k}}, \hat{x}_{\mathbf{k}^{\prime}}^{\dagger}\right]=\delta_{\mathbf{k}, \mathbf{k}^{\prime}}\right)$ :

$$
\left(\begin{array}{c}
\hat{x}_{i} \\
\hat{\Pi}_{x_{i}}
\end{array}\right)=\frac{1}{\sqrt{N}} \sum_{\mathbf{k}}\left(\begin{array}{c}
A_{\mathbf{k}}^{x} \\
i B_{\mathbf{k}}^{x}
\end{array}\right)\left[\hat{x}_{\mathbf{k}} e^{i \mathbf{k} \cdot \mathbf{r}_{i}} \pm \hat{x}_{\mathbf{k}}^{\dagger} e^{-i \mathbf{k} \cdot \mathbf{r}_{i}}\right] .
$$

Here, $A_{\mathbf{k}}^{x}=\sqrt{\hbar / 2 m_{x} \omega_{x}(\mathbf{k})}=B_{\mathbf{k}}^{x} / \omega_{x}(\mathbf{k}), N$ is the number of sites, and the "masses" are given by $m_{\sigma}=$ $\hbar^{2}|\log | \psi_{0}|| / 2 J z\left|\psi_{0}\right|^{2}$ and $m_{\varphi}=\hbar^{2} / U$. We finally obtain the quadratic Hamiltonian

$$
\hat{H}_{\mathrm{eff}}=\sum_{\mathbf{k}} \hbar \omega_{\varphi}(\mathbf{k}) \hat{\varphi}_{\mathbf{k}}^{\dagger} \hat{\varphi}_{\mathbf{k}}+\sum_{\mathbf{k}} \hbar \omega_{\sigma}(\mathbf{k}) \hat{\sigma}_{\mathbf{k}}^{\dagger} \hat{\sigma}_{\mathbf{k}}
$$

describing phase and amplitude fluctuations above the mean-field ground state. The dispersions of the modes are given by

$$
\begin{aligned}
\hbar \omega_{\sigma}(\mathbf{k}) & =\sqrt{U J z}\left|\psi_{0}\right|^{2} \\
& \times \sqrt{\left.32|\log | \psi_{0}\right|^{2}\left\{2+\log \left|\psi_{0}\right|[1+\gamma(\mathbf{k})]\right\} / u}, \\
\hbar \omega_{\varphi}(\mathbf{k}) & =\sqrt{U J z}\left|\psi_{0}\right| \sqrt{1-\gamma(\mathbf{k})},
\end{aligned}
$$

where $\gamma(\mathbf{k})=(2 / z) \sum_{l=1}^{d} \cos \left(\mathbf{k} \cdot \mathbf{a}_{l}\right)$ with lattice vectors $\mathbf{a}_{l}$. The amplitude mode is characterized by a finite gap $\Delta_{\sigma}=\hbar \omega_{\sigma}(0)$ extending throughout the entire range of parameters [13], cf. Fig. 22 for small $u \rightarrow 0$ the amplitude mode becomes nondispersive, i.e, $\hbar \omega_{\sigma}(\mathbf{k}) \approx \Delta_{\sigma}$ for all $\mathbf{k}$. The phase mode, on the other hand, is gap-less and characterized by a sound velocity $v_{s}^{\text {eff }}=\sqrt{U J z\left|\psi_{0}\right|^{2}} a / \hbar$. This is the Gross-Pitaevskii result, up to the factor $\left|\psi_{0}\right|^{2}$ accounting for the depletion of the condensate at large $u$.

Because the sound mode corresponds to a density fluctuation it can be probed directly by measuring the dynamic structure factor related to the density-density response of the system. This has been done in systems of ultracold atoms by using Bragg spectroscopy [14]. The gapped amplitude mode described above is not directly accessible to Bragg spectroscopy because it does not involve a density modulation. Rather, it is excited by perturbations that act to modulate the particle number variance, or equivalently, the distance to the Mott insulator phase. Experiments in ultracold atoms have done just that [9]. By modulating the strength of the optical lattice potential, those experiments effectively modulate the tunneling which is exponentially sensitive to the lattice depth. We remark that a similar modulation of the coupling was proposed to be relevant in experiments on Josephson junction arrays measuring the attenuation of ultrasound [15]; however, only coupling to the phase degrees of freedom was considered in this context.

To calculate the response to a lattice modulation, we return to the classical theory $\mathcal{L}_{\text {eff }}$, cf. Eq. (8), and extract the kinetic energy by replacing $J \rightarrow J+h$, keeping track of terms $\propto h$. Going through the re-quantization procedure (9), we obtain the kinetic-energy operator 


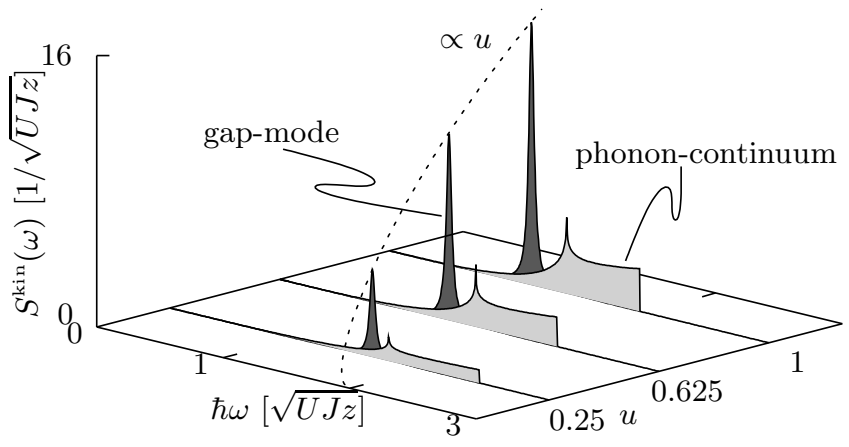

FIG. 3: The response function to the lattice modulation $S^{\text {kin }}(\omega)$ within the quadratic theory for different values of the interaction strength $u$ in two dimensions. The dark gray peak relates to the absorption of a single amplitude mode at $\hbar \omega=\Delta_{\sigma}$ (for illustrational purposes we give the delta-peak a finite width). The area in faint gray corresponds to the two-phonon continuum excited at energies below twice the phonon bandwidth $\hbar \omega \leq 2 W_{\varphi}$ (the logarithmic divergence is a density-of-states effect peculiar to two dimensions). Both absorption probabilities scale as $u$ at small $u$.

$\hat{T}=\hat{T}_{\sigma}+\hat{T}_{\varphi}$ which we expand to lowest order in $u$

$$
\begin{aligned}
& \hat{T}_{\sigma} \approx \sqrt{u} \frac{z}{2 \sqrt{2}} \sqrt{N}\left(\hat{\sigma}_{0}+\hat{\sigma}_{0}^{\dagger}\right), \\
& \hat{T}_{\varphi} \approx \sqrt{u} \sum_{\mathbf{k}} \frac{z}{4} \sqrt{1-\gamma(\mathbf{k})}\left[\hat{\varphi}_{\mathbf{k}} \hat{\varphi}_{-\mathbf{k}}+\hat{\varphi}_{\mathbf{k}}^{\dagger} \hat{\varphi}_{-\mathbf{k}}^{\dagger}+\hat{\varphi}_{\mathbf{k}}^{\dagger} \hat{\varphi}_{\mathbf{k}}\right] .
\end{aligned}
$$

The operator $\hat{T}_{\sigma}$ describes the expected direct coupling of the lattice modulations to the amplitude mode at $\mathbf{k}=0$. The other term $\hat{T}_{\varphi}$ describes pair-excitations of phase modes (phonons) by the lattice modulations. Both operators scale as $\sqrt{u}$ for small interactions.

Accordingly, the linear response function $S^{\mathrm{kin}}(\omega)=$ $\sum_{n}|\langle n|\hat{T}| 0\rangle|^{2} \delta\left(\hbar \omega-\hbar \omega_{n 0}\right)$, with $|n\rangle$ the eigenstates of the unperturbed Hamiltonian (10) and $\hbar \omega_{n 0}$ the energy differences between states $|0\rangle$ and $|n\rangle$, is composed of two parts including a single mode peak due to the gapped mode and a two-phonon continuum:

$$
S^{\mathrm{kin}}(\omega)=\frac{2 z^{2}\left|\psi_{0}\right|^{4}|\log | \psi_{0}||^{2}}{\sqrt{1+\log \left|\psi_{0}\right|}} N \delta\left(\hbar \omega-\Delta_{\sigma}\right)+S_{2 \mathrm{p}}^{\mathrm{kin}}(\omega) .
$$

Both terms scale as $u$, see Fig. 3. In two dimensions, the two-phonon continuum is given by $S_{2 \mathrm{p}}^{\text {kin }}(\omega) \propto$ $u \omega^{3} K\left(\hbar \omega \sqrt{1-\left(\hbar \omega / 2 W_{\varphi}\right)^{2}} / W_{\varphi}\right)$, with $K(x)$ the complete elliptic function; going to higher dimensions requires numerical evaluation.

Expanding the effective Lagrangian $\mathcal{L}_{\text {eff }}$ to third order in the fields $\sigma_{i}$ and $\varphi_{i}$ provides us with the most relevant decay channel of the amplitude mode which turns out to involve two counter-propagating phonons. We find that the small (in dimensions larger than 1) phase space for such a process leaves the mode under-damped and damping even becomes irrelevant in the limit $u \rightarrow 0$. Finally, we comment that the same results can be obtained using an RPA type calculation; such an approach allows for a systematic improvement of the above results and will be discussed in a future publication [16].

In summary, we derived a gapped amplitude (Higgstype) mode showing up in the quantum phase model using a dynamical variational approach and discussed its relevance in the context of superfluid bosonic atoms exhibiting strong correlations due to the presence of an optical lattice. Our analysis demonstrates that this mode persists down to weak coupling $u \approx 1 / \bar{n}^{2}$ where the Gross-Pitaevskii description takes over. We note that broken translation invariance (due to the presence of a lattice) is crucial for the existence of this mode. This is also reflected in the experiment where the coupling to this mode is introduced through a modulation of the lattice; while experiments have provided evidence for the presence of such a mode [1], their spectral resolution does not yet allow for its detailed analysis. Coupling to this mode in a Josephson junction array seems difficult due to the rigidity of the coupling parameters; on the other hand, the presence of a charge density wave in $\mathrm{NbSe}_{2}$ may give access to this mode [17, 18, 19]. While our analysis applies to the case of commensurate filling, we expect our results to remain valid away from this limit as the presence of the amplitude mode is connected with a squeezed Gaussian wave function and does not require the presence of particle-hole symmetry.

We thank F. Hassler and R. Barankov for insightful discussions and acknowledge financial support from the Swiss National Foundation through the NCCR MaNEP. S.H. thanks the Institut Henri Poincare - Centre Emile Borel for hospitality and support. E.A. was supported by the US-Israel binational science foundation.

[1] M. P. A. Fisher, P. B. Weichman, G. Grinstein, and D. S. Fisher, Phys. Rev. B 40, 546 (1989), URL http://link.aps.org/abstract/PRB/v40/p546

[2] M. Greiner, O. Mandel, T. Esslinger, T. W. Hänsch, and I. Bloch, Nature 415, 39 (2002), URL http://dx.doi.org/doi:10.1038/415039a.

[3] M. Kramer, C. Tozzo, and F. Dalfovo, Phys. Rev. A 71, 061602(R) (2005), cond-mat/0410122.

[4] M. A. Cazalilla, A. F. Ho, and T. Giamarchi, New J. Phys. 8, 158 (2006), URL http://www.iop.org/EJ/abstract/1367-2630/8/8/158/

[5] S. D. Huber, E. Altman, H. P. Büchler, and G. Blatter, Phys. Rev. B 75, 085106 (2007), URL http://link.aps.org/abstract/PRB/v75/e085106

[6] S. Sachdev, Quantum Phase Transitions (Cambridge University Press, 1999).

[7] S. Doniach, Phys. Rev. B 24, 5063 (1981), URL http://link.aps.org/abstract/PRB/v24/p5063.

[8] E. Simanek, Phys. Rev. B 22, 459 (1980), URL http://link.aps.org/abstract/PRB/v22/p459

[9] T. Stöferle, H. Moritz, C. Schori, M. Köhl, and T. Esslinger, Phys. Rev. Lett. 92, 130403 (2004), URL 
http://link.aps.org/abstract/PRL/v92/e130403

[10] R. Jackiw and A. Kerman, Phys. Lett. A $\quad \mathbf{7 1}, \quad 158 \quad$ (1979), URL http://dx.doi.org/doi:10.1016/0375-9601(79)90151-8

[11] C. Schori, T. Stöferle, H. Moritz, M. Köhl, and T. Esslinger, Phys. Rev. Lett. 93, 240402 (2004), URL http://link.aps.org/abstract/PRL/v93/e240402

[12] The Lambert- $W$ function is defined as the solution to $W(x) \exp [W(x)]=x$. It is real, negative, and monotonous for $x \in[-1 / e, 0]$ with $W(-1 / e)=-1$ and $W(0)=0$. Furthermore, $\mathrm{W}(x) \approx x$ for $x \rightarrow 0$.

[13] The gap vanishes at the spinodal $u_{\star}$, reflecting the consistency of the theory with both the critical theory [6] and previous studies of the amplitude mode [5].

[14] J. Stenger, S. Inouye, A. P. Chikkatur, D. M. Stamper-Kurn, D. E. Pritchard, and W. Ket- terle, Phys. Rev. Lett. 82, 4569 (1999), URL http://link.aps.org/abstract/PRL/v82/p4569.

[15] B. Muhlschlegel and D. L. Mills, Phys. Rev. B 29, 159 (1984), ISSN 0163-1829, URL http://link.aps.org/abstract/PRB/v29/p159

[16] S. D. Huber, E. Altman, and G. Blatter (2007), to be published.

[17] R. Sooryakumar and M. V. Klein, Phys. Rev. Lett. 45, 660 (1980), URL http://link.aps.org/abstract/PRL/v45/p660

[18] X. L. Lei, C. S. Ting, and J. L. Birman, Phys. Rev. B 32, 1464 (1985), URL http://link.aps.org/abstract/PRB/v32/p1464.

[19] C. M. Varma, J. Low Temp. Phys. 126, 901 (2002), URL http://dx.doi.org/10.1023/A:1013890507658 